\title{
„Und täglich grüßt das Murmeltier“ oder Selbsterfahrung in der Zeitschleife. Zur Musterbildung in Interaktionssystemen
}

\author{
Thomas Bachmann
}

Eingegangen: 16. März 2021 / Angenommen: 23. April 2021 / Online publiziert: 2. August 2021

(C) Der/die Autor(en) 2021

Zusammenfassung Anhand der Films „Und täglich grüßt das Murmeltier“ wird das Konzept der doppelten Kontingenz erklärt und die Entstehung von tragfähigen Beziehungen in Therapie, Coaching und Beratung untersucht. Dabei wird gezeigt, dass Resonanz in Beziehungen, jenseits von Tools und Methoden, eine wesentliche Quelle für Selbsterfahrung und Lernen darstellt.

Schlüsselwörter Beziehung $\cdot$ Selbsterfahrung $\cdot$ Resonanz $\cdot$ Doppelte Kontingenz

\section{"Groundhog day" or self-experience in the time loop. On pattern emergence in interaction systems}

\begin{abstract}
Based on the film "Groundhog Day", the concept of double contingency is explained and the emergence of sustainable relationships in therapy, coaching and consulting is examined. It is shown that resonance in relationships, beyond tools and methods, is an essential source for self-experience and learning.
\end{abstract}

Keywords Relationship - Self-experience $\cdot$ Resonance $\cdot$ Double contingency

\section{Handlung}

Phil Connors, Misanthrop und Zyniker, ist Wetterreporter aus Pittsburgh, der alle Jahre wieder vom „Groundhog Day“ am 2. Februar in der Kleinstadt Punxsutawney, Pennsylvania, berichten muss. Zusammen mit seinem Fernsehteam, der attraktiven

PD Dr. Thomas Bachmann $(\bowtie)$

Institut für Psychologie, Humboldt-Universität zu Berlin, Unter den Linden 6, 10099 Berlin,

Deutschland

E-Mail: bachmann.thomas@hu-berlin.de 
Aufnahmeleiterin Rita und Kameramann Larry, kommt er dort am Vortag entsprechend schlecht gelaunt an. Routiniert rasselt er seine Reportage herunter und möchte möglichst schnell wieder weg, was ein überraschend heraufgezogener Schneesturm verhindert, und so muss das Drehteam noch einmal in Punxsutawney übernachten.

Am nächsten Tag passiert etwas Überraschendes. Phil Connors erwacht wieder am Tag zuvor, dem „Murmeltiertag“ - der Radiowecker spielt „I Got You Babe“ -, und alles läuft wieder genau gleich ab. Erst kann er nicht fassen, was da passiert, fügt sich dann in sein Schicksal und liefert noch einmal seine Reportage ab. Er sucht Hilfe, doch niemand will ihm glauben oder kann ihm helfen. Auch der nächste Tag ist wieder ein 2. Februar usw. Phil beginnt schließlich die Situation zu akzeptieren und fängt an, damit zu experimentieren. Er fordert sein Schicksal heraus, wird zum Helden, zum Künstler, zum Kriminellen, versucht sich umzubringen. Und doch erwacht er am nächsten Morgen wieder am Tag zuvor.

Phil versucht nun jeden Tag, den er zwangsweise immer wieder genauso erleben muss und an dem immer wieder genau die gleichen Dinge passieren, so zu gestalten, dass er seine attraktive Kollegin Rita für sich gewinnen kann. Doch für Rita beginnt die Geschichte immer wieder von vorn, während sich Phil an die Tage zuvor erinnert und somit herausfinden kann, wer Rita ist und was sie mag und was nicht. Zu Beginn jedes Tages findet Rita Phil unsympathisch und arrogant, doch mit jedem Tag, den er sie besser kennenlernt, kann er ihre Wünsche und Ansichten besser erahnen. Und weil er jeden Tag wieder neu Anlauf nehmen kann, kommt er auch jedes Mal ein Stückchen weiter. Schließlich fühlt sich Rita so gut verstanden, als hätten sie sich aus einem früheren Leben gekannt. Immer besser gelingt es Bill innerhalb weniger Stunden, ihr Herz zu gewinnen, doch am Ende erwacht er trotzdem jeden Morgen wieder im gleichen Hotelbett, und der Radiowecker spielt den immer gleichen Song: „I Got You Babe“.

Erst als Phil seine Strategie aufgibt, nicht mehr taktiert und manipuliert, als er beginnt sich einzulassen, seine Absichten aufgibt, sich authentisch verhält und Gefühle zeigt, passiert das Wunder: Er erwacht neben Rita im Hotelbett, und der Radiowecker hat ihn diesmal nicht geweckt. Er hat die Zeitschleife verlassen. Phil und Rita beschließen, ihr künftiges Leben miteinander in Punxsutawney zu verbringen (Wikipedia 2021).

\section{Situation}

Der Film basiert auf unterhaltsame Weise auf dem Konzept der doppelten Kontingenz aus der Systemtheorie (Luhmann 1984). Bill und Rita sind füreinander kontingent. Zumindest was Bills Absicht betrifft, Rita zu verführen, herrscht unfassbare Unbestimmtheit. Sie mag ihn nicht und interessiert sich nicht für ihn. Er kennt sie nicht und hat für sein Vorhaben nur einen Tag Zeit. Das Interessante an diesem filmischen Gedankenexperiment ist die Idee, dass eine der beiden Personen immer wieder von vorn beginnen kann, die Interaktion zu gestalten und so die Beziehung zu entwickeln. Eine Person kann am Gegenüber lernen, experimentieren und Erfahrungen machen. Was sich sonst über Tage und Wochen hinzieht bzw. gar nicht erst stattgefunden hätte, weil man sich nicht mochte, oder was frühzeitig ab- 
gebrochen worden wäre, weil an einer Stelle das Falsche getan wurde, kann wie im Labor immer wieder unter gleichen Bedingungen wiederholt werden. Kann man es eigentlich schaffen, von jedem Menschen geliebt zu werden, wenn es nur lange genug Interaktionszeit gibt, sich kennenzulernen und sich aufeinander einzustellen? Welche Interaktionen sind förderlich, welche führen zum Abbruch? Welches Tempo ist das richtige?

An der wiederholten Interaktion mit der authentischen und offenherzigen Rita, die sich - bedingt durch die Zeitschleife - in ihrem Verhalten und ihrem Charakter nicht verändert, beginnt Phil zu wachsen und zu reifen. Zunächst geht er strategisch vor, versucht zu beeindrucken und zu imponieren. Dabei probiert er sich an verschiedenen Rollenklischees: Er spielt den Retter, den Helden, den Philanthropen, den guten Zuhörer oder den Kinderfreund. Er versucht jemand zu sein, der er nicht ist, in der Hoffnung, damit die Erwartungen der anderen und damit die von Rita zu treffen. Doch er scheitert jedes Mal und muss wieder von vorn beginnen und sich eine neue Strategie überlegen. Nach und nach beginnt er zu verstehen, dass nicht das strategische Verhalten der Schlüssel zu Rita und damit zum Verlassen der Zeitschleife ist, sondern die authentische und absichtslose Begegnung.

Luhmann (1984) führt aus, dass es die „doppelte Kontingenz“ ist, weshalb wir Menschen Kommunikationssysteme erschaffen (müssen). Das gemeinsam erschaffene Muster aus Kommunikation und Anschlusskommunikation schafft Erwartbarkeit, reduziert Unsicherheit (und damit Komplexität) und lässt eine gemeinsame Wirklichkeit und Sinn und damit koordiniertes Handeln entstehen. Das zirkuläre Miteinander, das Sich-aneinander-entwickeln und Ko-kreieren durch Kommunikation, führt zur Emergenz dessen, was wir Beziehung nennen. Das Entstehen dieses Musters lässt sich nicht steuern, allenfalls beeinflussen. Das ist der Grund, weshalb wir uns nicht mit jedem Menschen, den wir treffen, auf Anhieb verstehen. Es braucht den richtigen Moment, und es muss zwischen uns passen, damit das Spiel aus Kommunikation und Anschlusskommunikation in Gang kommt und seine Eigendynamik entwickelt. Luhmann geht sogar noch weiter und bezeichnet solche Kommunikationssysteme als autopoietisch. Sie erschaffen sich selbst, aus sich heraus, suchen sich ihren eigenen Weg, selbstreferentiell und operational geschlossen.

Im Film erfährt Phil jeden Tag aufs Neue den Unterschied zwischen Beeinflussbarkeit und Steuerbarkeit. Seine Taktiken der Beziehungsgestaltung funktionieren jeweils nur bis zu einem gewissen Punkt. Wenn genug Komplexität entstanden ist, nimmt die Eigendynamik des Systems die Zügel in die Hand. Phil ist dabei nur der Initiator oder Anschieber, ab einem bestimmten Punkt lässt sich die weitere Entwicklung nicht mehr steuern, denn zu viele Möglichkeiten sind entstanden und können in Sackgassen führen. Das liegt vor allem daran, dass die Beziehung zwischen Phil und Rita nicht auf einem ko-kreierten System basiert, sondern von Phil taktischmanipulativ hergestellt wurde. Und so kommt es bei der kleinsten Irritation jedes Mal zum Abbruch. Die Anschlusskommunikation bleibt aus. Ende der Autopoiesis.

Phil lernt daraus. Er lernt das, was er nur von Rita lernen kann, denn sie verkörpert Offenheit, Menschlichkeit, Herzlichkeit und Empathie, sie ist neugierig, authentisch und zugewandt. Auf der Suche nach dem Schlüssel zu Rita findet er den Schlüssel zu sich selbst, und dabei gelingt es ihm mehr und mehr, sich einzulassen und unvoreingenommen zu sein, Arroganz, Zynismus und Ironie zu überwinden und sich 
der Welt mit dem Herzen zuzuwenden. Phil beginnt sich selbst zu erfahren. Und das Schöne ist, dass er das jeden Tag von Neuem ausprobieren kann, ohne Schaden zu nehmen, ohne dass es jemand übel nehmen könnte. Besser hätte man Bubers berühmten Satz „Der Mensch wird am Du zum Ich“ (Buber 1995) filmisch nicht illustrieren können: das „Du“ als Spiegel, als Feedbackquelle, als Resonanzboden, das ,Zwischen“ als Erfahrungsraum, die Beziehung, wenn sie trägt, als geronnene Begegnungen.

\section{Intervention}

Wie lässt sich die beraterische Intervention beschreiben, die in diesem Film steckt? Ist es die Wiederholung des Immergleichen und das dadurch erzwungene Trialand-Error-Lernen von Phil? Aus meiner Sicht ist Rita die „Intervention“. Sie ist das Instrument, welches Phil zum Klingen bringen möchte. Dabei ist sie völlig absichtslos. Sie ist einfach nur da, so wie sie ist, und was sie zur Verfügung stellt, ist Resonanz (Rosa 2016), nicht mehr und nicht weniger.

\section{Transfer}

Aus vielen Untersuchungen zur Wirksamkeit von Therapie, Coaching oder Pädagogik weiß man, dass die Beziehung der entscheidende Faktor ist (vgl. Bachmann 2018; Jansen et al. 2004; Grawe et al. 1994; Hattie 2003). Methoden, Settings, Tools oder Formate unterliegen dem Primat der Beziehung. Welches dabei die ausschlaggebenden Beziehungsqualitäten sind, ist nur teilweise bekannt und der empirischen Forschung nicht vollständig zugänglich.

Wie muss die Beziehung zu einer anderen Person sein, sodass ich an (nicht von!) ihr lernen kann? Was muss mein Gegenüber tun oder lassen, damit ich mit mir Lernerfahrungen machen kann? Aus der Rolle von Rita ließen sich einige Eigenschaften und Verhaltensweisen ableiten, welche ihr Verhalten in besonderem Maße kennzeichnen:

- offen, unvoreingenommen, interessiert

- authentisch, d.h. stimmig, glaubwürdig, echt

- integer, d.h. unbescholten (nicht taktisch oder manipulativ agierend)

- wertebasiert

- unabhängig

Von allen gängigen Therapierichtungen unterscheidet sich die Gestalttherapie darin, wie die helfende Beziehung gestaltet und mit und durch sie der therapeutische Prozess herbeiführt wird. Dabei geht es darum, während der Therapie Beziehungserfahrungen zu machen, zu erleben, zu fühlen und zu verstehen. Das wichtigste Mittel ist dabei die unmittelbare Resonanz des Therapierenden bezüglich des Kontaktverhaltens seines Gegenübers. Resonanz geben heißt dabei, im Mitschwingen mit dem anderen das eigene Erleben mitzuteilen, so, wie es in diesem Moment gerade ist, das, was ich in der Begegnung mit meinem Gegenüber gerade fühle, wie es mir 
mit ihm geht, was es in mir auslöst, was in mir gerade passiert. Damit entsteht eine grundlegende Beziehungserfahrung, die das Selbst und seine Beziehungsfähigkeit stärkt. Praktizierende der Gestalttherapie muten sich ihrem Gegenüber in besonderer Weise zu, zeigen sich mit ihrem Erleben, authentisch und spontan, und doch reflektiert und prozessorientiert. Dabei kommt ihnen gleichzeitig die Aufgabe zu, „das Dazwischen zu hüten“ (Hycner 2003), also Gestaltende jenes Erfahrungsraum zu sein, der es ermöglicht, sich authentisch zu begegnen, ohne zu stark zu irritieren.

Veränderung, Lernen, Entwicklung und Klärung in Therapie, Coaching und Beratung braucht neben Methoden und Tools vor allem Menschen, die sich trauen, anderen zu begegnen und sich selbst als Instrument für Lernen in Beziehung zur Verfügung stellen.

\author{
Originaltitel: Groundhog Day \\ Erscheinungsjahr: 1993 \\ Land: USA \\ Regie: Harold Ramis \\ Besetzung: Bill Murray, Andie MacDowell, Chris Elliott u.a. \\ Verfügbarkeit: Amazon: DVD, Amazon prime, Netflix: Streaming
}

Funding Open Access funding enabled and organized by Projekt DEAL.

Open Access Dieser Artikel wird unter der Creative Commons Namensnennung 4.0 International Lizenz veröffentlicht, welche die Nutzung, Vervielfältigung, Bearbeitung, Verbreitung und Wiedergabe in jeglichem Medium und Format erlaubt, sofern Sie den/die ursprünglichen Autor(en) und die Quelle ordnungsgemäß nennen, einen Link zur Creative Commons Lizenz beifügen und angeben, ob Änderungen vorgenommen wurden.

Die in diesem Artikel enthaltenen Bilder und sonstiges Drittmaterial unterliegen ebenfalls der genannten Creative Commons Lizenz, sofern sich aus der Abbildungslegende nichts anderes ergibt. Sofern das betreffende Material nicht unter der genannten Creative Commons Lizenz steht und die betreffende Handlung nicht nach gesetzlichen Vorschriften erlaubt ist, ist für die oben aufgeführten Weiterverwendungen des Materials die Einwilligung des jeweiligen Rechteinhabers einzuholen.

Weitere Details zur Lizenz entnehmen Sie bitte der Lizenzinformation auf http://creativecommons.org/ licenses/by/4.0/deed.de.

\title{
Literatur
}

Bachmann, T. (2018). Failure in coaching. Between craft and the art of creating relationships. In S. Kunert (Hrsg.), Failure management. Wiesbaden: Springer VS.

Buber, M. (1995). Ich und Du. Stuttgart: Reclam.

Grawe, K., Donati, R., Bernauer, F., \& Donati, R. (1994). Psychotherapie im Wandel: von der Konfession zur Profession. Göttingen: Hogrefe.

Hattie, J. (2003). Teachers make a difference: what is the research evidence? https://research.acer.edu.au/ research_conference_2003/4. Zugegriffen: 25. Apr. 2021.

Hycner, R. (2003). Für eine dialogische Gestalttherapie. In E. Doubrawa \& F.-M. Staemmler (Hrsg.), Heilende Beziehung: Dialogische Gestalttherapie (S. 59-82). Köln: gikPress.

Jansen, A., Mäthner, E. \& Bachmann, T. (2004). Erfolgreiches Coaching. Wirkfaktoren im Einzel-Coaching. Kröning: Asanger.

Luhmann, N. (1984). Soziale Systeme: Grundriss einer allgemeinen Theorie. Frankfurt/M.: Suhrkamp.

Rosa, H. (2016). Resonanz: Eine Soziologie der Weltbeziehung. Berlin: Suhrkamp. 
Wikipedia (2021). Und täglich grüßt das Murmeltier. https://de.wikipedia.org/wiki/Und_t\%C3\%A4glich_ gr\%C3\%BC\%C3\%9Ft_das_Murmeltier. Zugegriffen: 28. Febr. 2021.

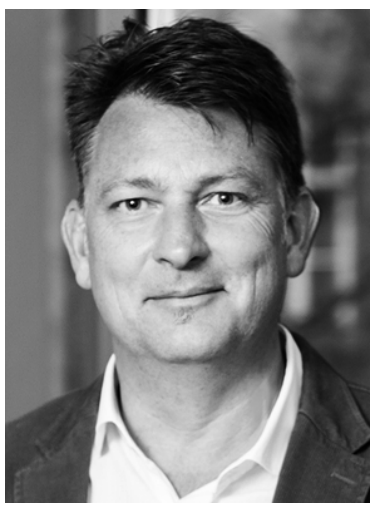

PD Dr. Thomas Bachmann Dipl.-Psych., Gründungsmitglied und Partner der artop GmbH; Studium der Arbeits- und Organisationspsychologie, Klinische Psychologie sowie Informatik, wissenschaftl. Mitarbeiter am Lehrstuhl für Methodenlehre und Statistik, 1997 Promotion in der Kognitiven Psychologie, 2019 Habilitation u. a. zu Systemtheorie und Gestalttherapie. Seit 1993 Berater, Trainer und Coach (DBVC, ICF), seit 2001 Ausbilder und Lehrtrainer für Coaches und Berater. 